\title{
Morphological features of elongated-anisotropic magnetosome crystals in magnetotactic bacteria of the Nitrospirae phylum and the Deltaproteobacteria class
}

\author{
Christopher T. Lefèvre , Mihály Pósfai , Fernanda Abreu , Ulysses Lins , \\ Richard B. Frankel , Dennis A. Bazylinski
}

\begin{abstract}
A B S T R A C T
High resolution transmission electron microscopy was used to study the crystallographic habits of the elongated magnetite crystals, variously described as bullet-, tooth- or arrowhead-shaped, in two recently described, uncultured, magnetotactic bacteria belonging to the Nitrospirae phylum designated Candidatus Magnetoovum mohavensis strain LO-1, and Candidatus Thermomagnetovibrio paiutensis strain HSMV-1; and a cultured sulfate-reducing magnetotactic bacterium of the Deltaproteobacteria class of the Proteobacteria phylum designated strain AV-1. The elongation axes of the magnetosomes do not coincide with the easy magnetization axis (which is [111]) but they are parallel to [100] in LO-1 and AV-1 and parallel to [110] in HSMV-1. In all three strains, magnetosome magnetite crystals appear to elongate at constant width, resulting in asymmetric shapes. Idealized crystal morphologies are proposed. Neither the control mechanism over crystal growth, nor the adaptiveness, if any, of such unusual crystal habits are known at the moment. Since similar elongated and asymmetric morphologies are unknown in inorganically-formed magnetite crystals, these forms of magnetosome magnetite appear to be excellent biomarkers.
\end{abstract}

\section{Introduction}

Magnetotactic bacteria (MTB) biomineralize magnetosomes comprising nano-scale magnetic-iron-mineral crystals enclosed in lipid bilayer membranes (Bazylinski and Frankel, 2004). The iron minerals are magnetite, $\mathrm{Fe}_{3} \mathrm{O}_{4}$, and greigite, $\mathrm{Fe}_{3} \mathrm{~S}_{4}$. A striking feature of magnetosome magnetite crystals is that they have different, consistent, twodimensional (2D) projected shapes in different bacterial species or strains when observed by transmission electron microscopy (TEM). The 2D projected shapes include cuboidal, quasi-rectangular and asymmetric (arrowhead-, tooth-, or bullet-shaped). The asymmetric crystal morphologies appear to be inconsistent with the cubic point symmetry $(\mathrm{m} 3 \mathrm{~m})$ of the spinel-type structure of magnetite.
While three-dimensional (3D) crystal habits cannot be determined from 2D projected images alone, idealized 3D crystal habits, based on low-index crystallographic forms, have been proposed (e.g., Lins et al., 2005; Mann et al., 1984; Matsuda et al., 1983; Meldrum et al., 1993a, 1993b). These habits include cuboctahedral, elongated-prismatic and elongated-anisotropic, for the corresponding cuboidal, quasirectangular and asymmetric $2 \mathrm{D}$ projected shapes, respectively. In this context, anisotropic means a crystal habit lacking a center of inversion symmetry. The overall sizes of the crystals, the width/length ratios, and the relative sizes of corner faces can vary from one species or strain of MTB to another, resulting in the distinctive projected shapes.

All MTB known to date are affiliated with the Alpha-, Gamma- and Deltaproteobacteria classes of the Proteobacteria phylum, or the Nitrospirae phylum. While magnetite-bearing MTB occur in all four taxa, greigite-bearing bacteria examined to date are phylogenetically affiliated with only the Deltaproteobacteria. Of the magnetite-bearing MTB, magnetosomes with cuboctahedral and elongated-prismatic idealized habits, respectively, occur only in those phylogenetically affiliated with the Alpha- and Gammaproteobacteria, (e.g., Magnetospirillum species, and Candidatus Magnetococcus marinus strain MC-1). On the other hand, magnetosomes with elongated-anisotropic habits have been found in MTB affiliated only with the Nitrospirae and the Deltaproteobacteria, (e.g., Candidatus Magnetobacterium bavaricum and Desulfovibrio magneticus strain RS-1, respectively). 
The most common 2D projected image of elongated-anisotropic crystals is the bullet or flat-top shape (fts), with one flat end and one narrower, rounded, end (Blakemore et al., 1980; Mann et al., 1987a, 1987b; Thornhill et al., 1994). Sometimes the magnetosome crystals with fts projections are bent in one direction along their length (Hanzlik et al., 2002). Fts magnetosome crystals in an uncharacterized MTB from the Exeter River (ER) in New Hampshire, USA were proposed to have an idealized, six-sided prismatic, magnetosome habit comprising four $\{111\}$ and two $\{100\}$ faces capped by two faces of $\{111\}$ with associated $\{111\}$ and $\{100\}$ corner faces. Crystal growth of a nascent cuboctahedron supposedly commences via nucleation on the magnetosome membrane and continues until the width of the crystal is about $40 \mathrm{~nm}$. Subsequent elongation then occurs parallel to $<112>$ while the crystal width remains relatively constant (Mann et al., 1987a, 1987b).

Some elongated-anisotropic magnetosomes have distinctive projected images with double-triangle shape (dts), two isosceles triangles sharing a common base. These dts magnetosomes occur in some MTB phylogenetically affiliated with the Nitrospirae and with the Deltaproteobacteria (Li et al., 2010; Lins et al., 2007; Pósfai et al., 2006; Vali and Kirschvink, 1990). Both projected triangles have the same width, but in mature crystals one triangle is longer than the other. Until now, no idealized habit for dts magnetosomes has been proposed.

Here we report on the idealized crystal habits and crystal size distributions for elongated-anisotropic magnetosome crystals in a newly cultivated MTB affiliated with the Deltaproteobacteria, and in two newly described, uncultured MTB affiliated with the Nitrospirae. The former organism, designated strain AV-1, appears to be a magnetotactic strain of Desulfonatronum thiodismutans (Pikuta et al., 2003) and was isolated from an ambient-temperature, alkaline spring in the Amaragosa Valley, near Death Valley Junction, California. The organism is helical in shape and possesses a single polar flagellum. Strain AV-1 contains dts magnetosomes arranged in single, or in some cells double, chains that traverse the length of the cell (Lefèvre et al., 2011a). One of the Nitrospirae, designated Candidatus Magnetoovum mohavensis strain LO-1, was collected from sediments of Lake Mead, Nevada, and contains dts magnetosomes arranged in three chains that resemble twisted braids (Lefèvre et al., 2011b). Since dts magnetosomes occur in strains AV-1 and LO-1, the idealized crystal habit for strain AV-1 also applies to strain LO-1. The other Nitrospirae, designated Candidatus Thermomagnetovibrio paiutensis strain HSMV-1, is a moderately thermophilic vibrio that was collected from a series of thermal springs in northern Nevada. Each cell contains fts magnetosomes arranged in a single chain (Lefèvre et al., 2010).

\section{Methods}

Details of the collection of cells of strains HSMV- 1 and LO- 1 from the environment and the isolation of strain AV-1 in pure culture are discussed in Lefèvre et al. (2010, 2011a and 2011b, respectively). Living cells of each type from cultures in late exponential to early stationary phase were deposited on carbon-coated grids and dried in air. The grids were examined as soon as possible after drying. Typically this time period ranged from an hour or two to several days. Grids were stored in small plastic vials under oxygen-free nitrogen to prevent oxidation of magnetite. Low magnification images and high resolution TEM (HRTEM) images were taken with a JEOL Model 3010 TEM at $300 \mathrm{kV}$. Tilt series of low magnification images and selected-area electron diffraction patterns (SAED) were taken with a Philips Model CM20 at $200 \mathrm{kV}$. To obtain SAED patterns we used a projected aperture with a diameter of $250 \mathrm{~nm}$ in the image plane.

Crystal habits were modeled with KrystalShaper software. We used the cubic point group $\mathrm{m} 3 \mathrm{~m}$, and variables included the faces present (with specific Miller-indices) and their distances from the center of the crystal. Magnetosome sizes were measured with ImageJ software (U.S. National Institutes of Health).

\section{Results}

3.1. Idealized crystal habits for dts magnetosomes in strains AV-1 and LO-1

TEM images of magnetosome chains in cells of strains AV-1, LO-1, and HSMV-1 are shown in Figs. $1 \mathrm{~A}-1 \mathrm{C}$, respectively. The dts magnetosomes in strain AV-1 appear to consist of two triangles: a short "base" triangle and an elongated triangle, with a common side. In small crystals $(<45 \mathrm{~nm})$ the two triangles are congruent. Whereas the sides of the base triangle are low-index crystal faces (see below), the sides of the elongated triangle are slightly curved. Such curved sides can only result from either the presence of a large number of high-index faces or some irregular surfaces that cannot be described by crystallographic faces (and thus by Miller-indices). In fact, the former case converges into the latter, since the more high-index faces appear on a crystal, the less regular the shape becomes.

Concerning the base triangle, the interpretation of the 3D shape is straightforward. The measured angle between the two sides that meet to form the tip in the projected image ranges from 70 to $90^{\circ}$ (Fig. 1A), exactly the two extreme values obtained by tilting a regular octahedron from [110] into [100] orientation, respectively. In other words, the projection of any two sides that join in the same vertex of a regular octahedron must be between 70 and $90^{\circ}$, as long as the octahedron is tilted about a $<100>$ axis. This also applies to slightly "distorted" or "elongated" octahedra in which the different $\{111\}$ faces are not equally developed, because the sizes of the faces do not affect the angles between them.

The above reasoning is confirmed by HRTEM images. The crystallographic directions in the HRTEM image of a single crystal in Fig. 2 can be clearly identified from both the Fourier transform and the lattice spacings. The magnetosomes are elongated along [100]. Since the sides of the projected base triangle coincide with $\{111\}$ octahedral faces, the base section of each magnetosome crystal is just half a regular octahedron. The presence of narrow (110)-type faces between the octahedral faces cannot be excluded on the basis of the HRTEM images. In strain LO-1, dts magnetosomes crystals are also elongated along [100] (Fig. 1B).

In order to understand the 3D shape of the elongated section of the dts magnetosome, images of a magnetosome chain in strain AV- 1 were sequentially acquired as the microscope stage was tilted as far as possible $\left( \pm 45^{\circ}\right)$. Although no magnetosome chain was entirely parallel to one of the tilt axes, the one shown in Fig. 3 was close. The approximate direction of the tilt axis is marked in the third panel. This tilt series indicates that the elongated section essentially has $\infty$-fold symmetry about the [100] elongation axis. This means that the habits of dts magnetosomes can be modeled simply on the basis of any two-dimensional projection. As discussed above, the base section is either a regular or a slightly distorted half-octahedron (four-sided pyramid). The elongated, pointed section consists of a series of high-index faces, mostly of \{hh1\}and \{h01\}-type forms (Fig. 4B). If faces of several such forms are present, the outline of the projected crystal appears to be curved. While there are many variations of forms that can be used to approach the curved outline of the crystals, the sizes of these faces cannot be varied randomly (if some are enlarged, faces of other forms disappear), so the length of the crystal is almost fixed for a given curvature. On the very elongated magnetosomes, $\{110\}$ and $\{100\}$-type faces, which are parallel to the elongation axis, are prominent close to the base. In this case, the crystal can be of any length. Of course, the model shown in Fig. 4 is an idealization of the magnetosome habit. In reality, it is possible that the surfaces of the elongated section are really curved and do not exactly correspond to crystallographic faces.

In addition to the dts magnetosomes, there are some magnetosomes with unusually curved $2 \mathrm{D}$ projections in strain AV-1 (see the arrowed magnetosomes in Fig. 1A). These crystals invariably show heterogeneous contrast in bright-field images, suggesting that they 

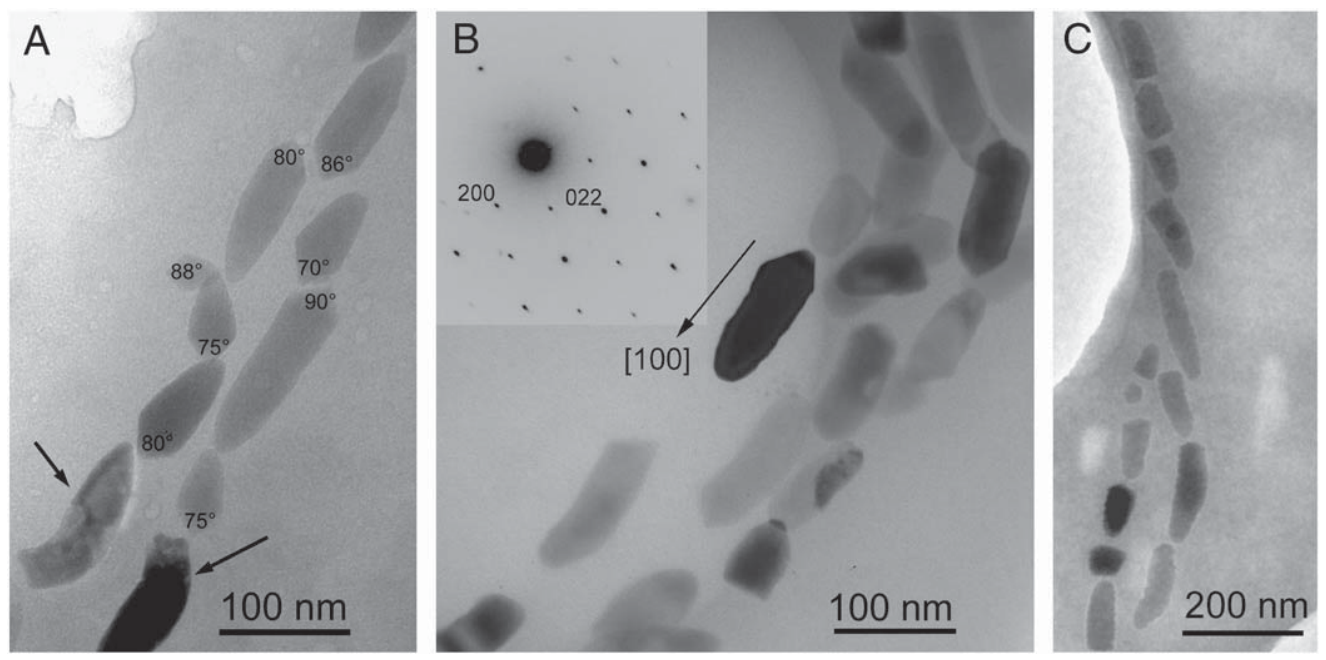

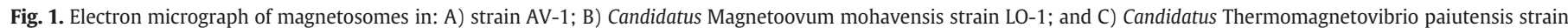

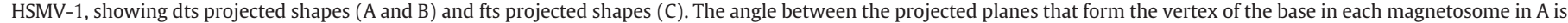

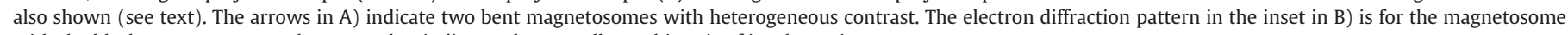
with the black contrast next to the arrow that indicates the crystallographic axis of its elongation.

are aggregates of multiple small crystals. The image in Fig. 5, from one of the curved magnetosomes, indicates that it consists of several crystallites that are misaligned with respect to each other. The crystallite on the right side and a small crystallite on the far left side are almost in a zone-axis orientation, whereas the other crystallites are in different orientations. On the basis of their contrasts and diffraction patterns, the aggregated crystals do not appear to be twinned. It is interesting that even the multi-crystalline, curved magnetosomes have elongated, pointed ends; however, their shapes are too complex to interpret from such images.

In summary, the elongated-anisotropic magnetosomes in strains AV-1 and LO-1 have the following features: i) the majority of magnetosome crystals have dts projected images and are single crystals without defects or twinning; a minority are strongly curved and comprise several crystallites that are not all in the same orientation; ii) the habits of the dts magnetosomes can be modeled on the basis of a regular, or slightly elongated, half-octahedron (four-sided pyramid) as a base, and an elongated, pointed section that consists mostly of high-index faces. The curved outlines suggest that the surface of the elongated section cannot be completely described using Miller-indices; iii) the axis of elongation of the dts magnetosomes is parallel to [100].

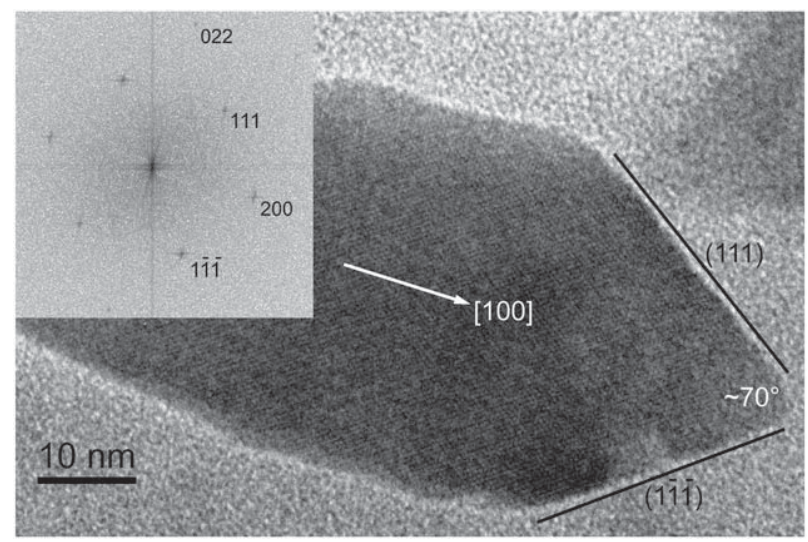

Fig. 2. A high resolution transmission electron micrograph (HRTEM) of a portion of a magnetosome from strain AV-1. Inset: the FFT corresponding to the lattice image. The white arrow indicates the axis of elongation. The indices of the faces of the base octahedron that are seen edge-on and the projected angle between them are also indicated.

\subsection{Idealized crystal habits for fts magnetosome crystals in HSMV-1}

A cell of strain HSMV-1 is shown in Fig. 1C and magnetosomes at higher magnification are shown in Fig. 6. The magnetosomes are elongated, with a nearly flat end and a narrow, rounded end. The rounded ends all point more or less in the same direction. A striking feature is that most of the magnetosomes are bent (e.g., 3, 4, and the unmarked magnetosome between them), somewhere along their length. Nevertheless, high-resolution images from all the numbered crystals in Fig. 6 show them to be single crystals of magnetite. Neither twin boundaries, nor other defects can be observed that would explain the bending.

A sequence of three images over a tilt range of $60^{\circ}$ of a chain of fts magnetosomes is shown in Fig. 7. The chain was approximately parallel to the tilt axis of the specimen stage (as indicated by the arrow in the third panel). The parallel or anti-parallel orientations of the rounded ends of the crystals appear to be random with respect to the chain in this cell. The edges of the crystals are wavy as if they were bound by irregular surfaces instead of proper crystal faces. Some look straight in the first panel of the sequence (e.g., B and C), but when rotated by $60^{\circ}$, are seen to be bent. Magnetosome $A$ appears
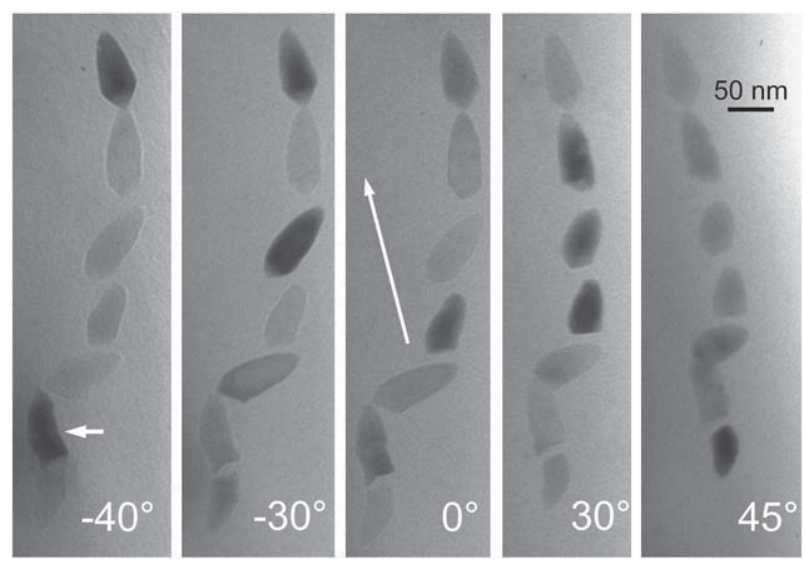

Fig. 3. Electron micrographs of a tilt series of a magnetosome chain in a cell of strain $A V-1$. The white arrow in the central panel indicates the direction of the tilt axis, and the angle of tilt is shown at the bottom of each panel. The small arrow in the left panel marks a curved, composite magnetosome. 

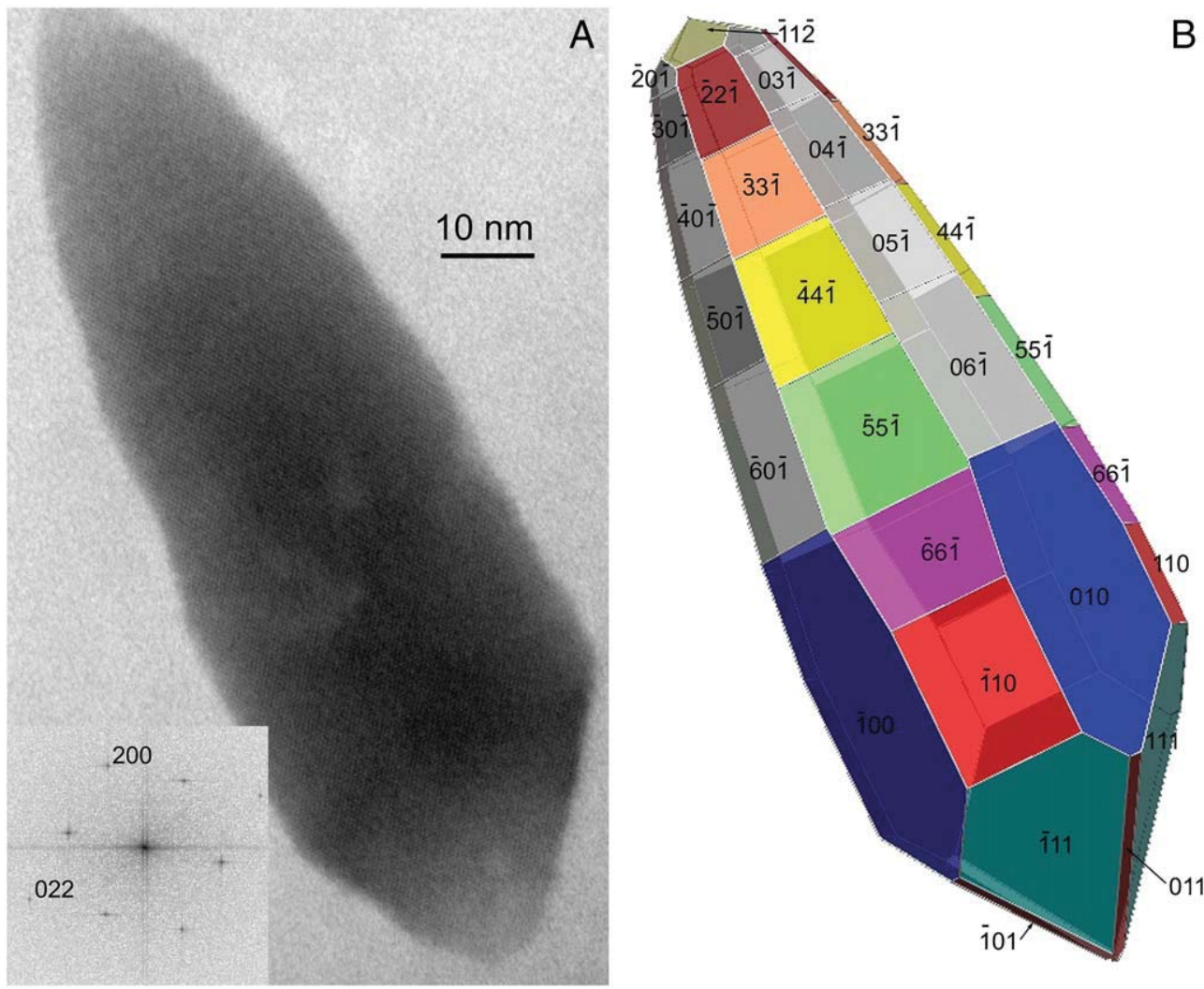

Fig. 4. A) HRTEM image and its inserted FFT of a dts-magnetosome in strain AV-1 and B) the idealized morphology of the crystal.

bent in the first panel but straight in the last panel. Some magnetosomes look straight in all three images.

A high-resolution image of the narrow, rounded end of magnetosome 2 in Fig. 6 along the [111] zone axis is shown in Fig. 8. The crystal is elongated in one of the $<110>$ directions. The flat end (not shown) is not a single face but an irregular or curved surface that can only be approximated with a $\{110\}$ plane. The long, prismatic sides of the crystal are bound by faces that are parallel to the [110] zone axis.

A tentative idealized morphology is shown in Fig. 9A. In this model the "prismatic" side faces include two faces of $\{100\}$, two of $\{110\}$ and four of $\{112\}$. Large $\{112\}$-type side faces were included because straight sides appear to coincide with faces of $\{112\}$. The capping faces on the rounded ends consist of a mixture of $\{100\},\{110\}$ and $\{111\}$. This is just one of many possible idealized models. For example, models with no $\{112\}$-type side faces but comprising only $\{100\},\{110\}$ and $\{111\}$ faces are also possible (Fig. 9B). Several high-index faces can also be added to the models to make the crystal gradually narrow to the rounded end as seen in Fig. 8 .

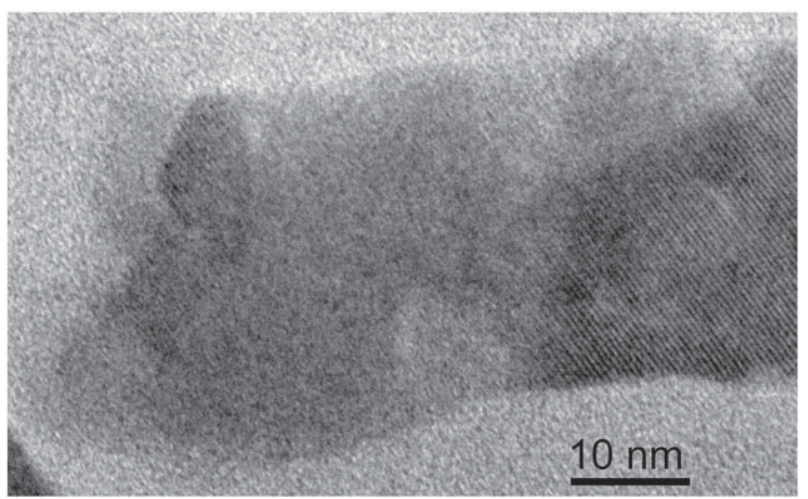

Fig. 5. HRTEM image of part of a curved magnetosome from strain AV-1 showing differential contrast, indicating that the magnetosome is an aggregate of several crystals.
In summary, the bent, elongated-anisotropic, fts magnetosomes in strain HSMV-1 have the following features: i) based on the measured crystallographic parameters, all crystals appear to be magnetite; ii) the magnetosomes are highly elongated and many of them are

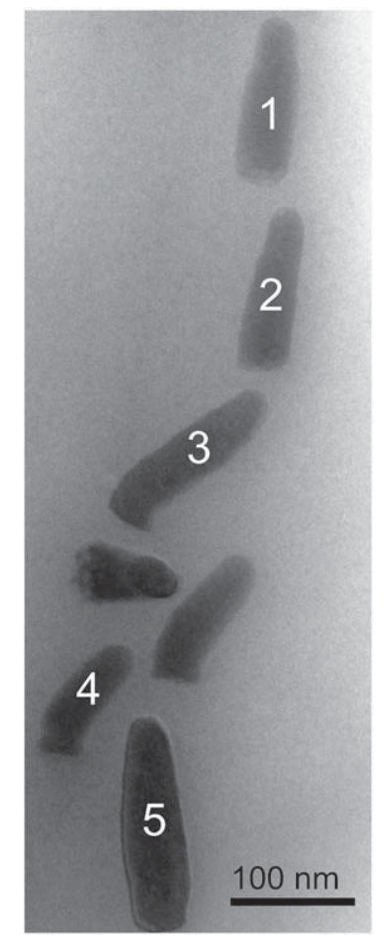

Fig. 6. Fts elongated-anisotropic magnetosomes in Candidatus Thermomagnetovibrio paiutensis strain HSMV-1. High-resolution structure images were obtained from each of the numbered crystals. 

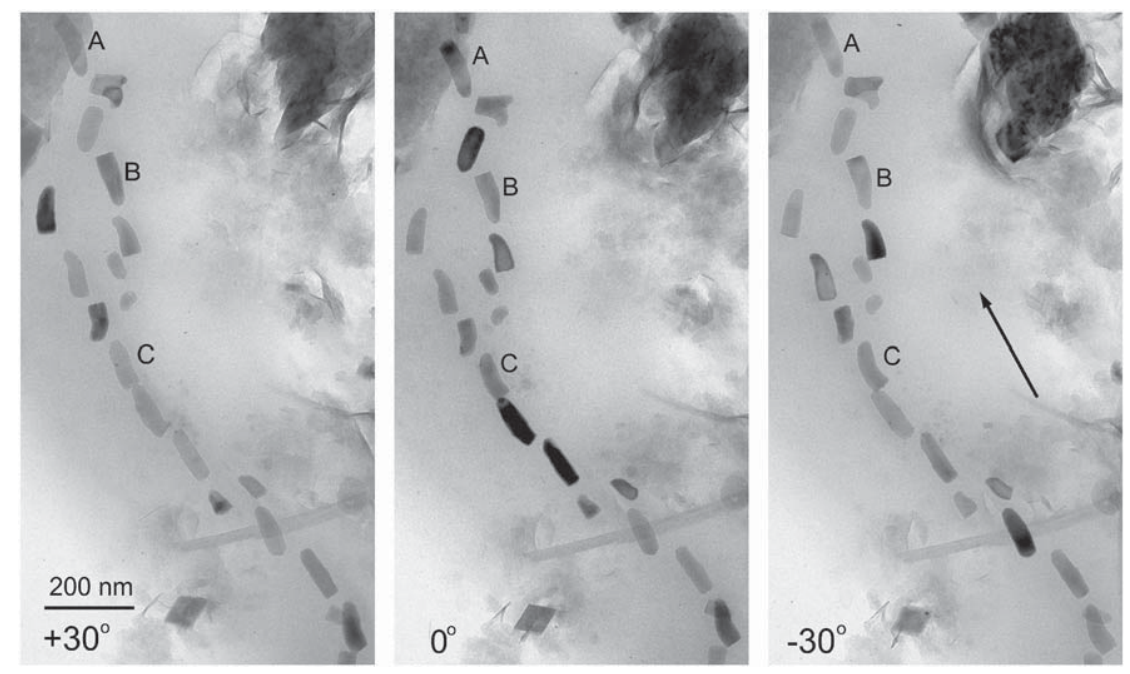

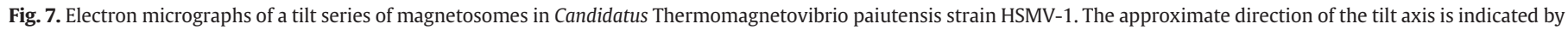
the arrow in the right panel, and the angle of tilt is shown at the bottom of each panel. Magnetosomes marked A, B, and C are discussed in detail in the text.

bent in one direction (hook-shaped); iii) from the analysis of high resolution images and their Fourier transforms obtained from five magnetosomes, the principal elongation axis is [110]; iv) idealized morphological models have elongated "prismatic" side faces that are parallel to [110] and may include certain faces of the $\{100\},\{111\}$, $\{110\}$, and $\{112\}$ forms. The narrow, rounded ends of the models consist of faces of the same forms. However, it should be noted that the outlines of the crystals are irregular, and any model is just an approximation. The crystals do not appear to be bound by well-developed, smooth, faces.

\subsection{Crystal-size distributions}

The crystal size distributions (CSD) and width as a function of length plots are shown for strains AV-1, LO- 1 and HSMV-1 in Fig. 10. The CSD is negatively skewed for strain AV-1, while the other two CSDs are closer to Gaussian. In all cases, the crystal width saturates at about $45 \mathrm{~nm}$ and remains constant as the length increases from about $45 \mathrm{~nm}$ to over $200 \mathrm{~nm}$. These results are consistent with those for ER and other uncharacterized MTB (Isambert et al., 2007; Li et al., 2010; Mann et al., 1987a, 1987b).

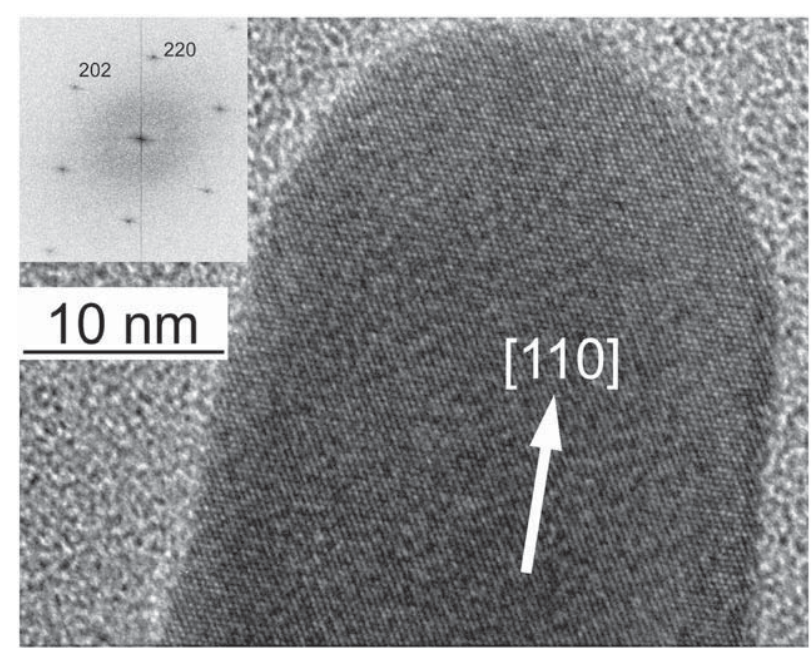

Fig. 8. High resolution transmission electron micrograph of the rounded end of $\mathrm{fts}$ magnetosome 2 in Fig. 6, with its FFT in the inset. The white arrow indicates the axis of elongation parallel to [110].

\section{Discussion}

In the MTB phylogenetically affiliated with the Alphaproteobacteria, magnetosomes arranged in a chain are invariably oriented with a $<111>$ crystal axis parallel to the chain axis. In those strains with elongated-prismatic habits, the axis of elongation is the $<111>$ axis of orientation. Based on what we report here, this is not the case for the elongated-anisotropic magnetosomes in MTB affiliated with either the Nitrospirae or the Deltaproteobacteria. While elongated-anisotropic magnetosomes are usually oriented with their long axes parallel to the chain axis, the axis of elongation can vary among $<100>,<110>$, $<111>$, and $<112>$.

The easy (lowest energy) direction of magnetization in single crystals of magnetite is parallel to $<111>$, whereas directions parallel to $<110>$ and to $<100>$ are intermediate and hard (highest energy), respectively. At first glance, it may be surprising that the magnetite crystal habits with dts projections and fts projections are elongated parallel to $\langle 100>$ and $<110>$, respectively. However, magneto-
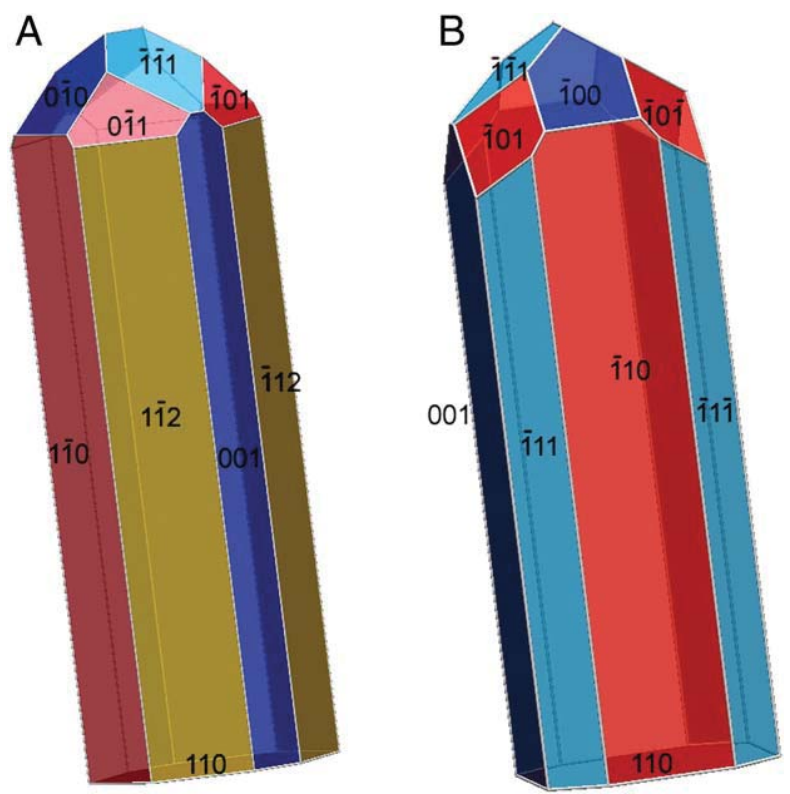

Fig. 9. Two of many possible morphological models for the fts magnetosome crystals in Candidatus Thermomagnetovibrio paiutensis strain HSMV-1. Both are elongated parallel to [110]. The models do not include the bending of the magnetosome crystals. 

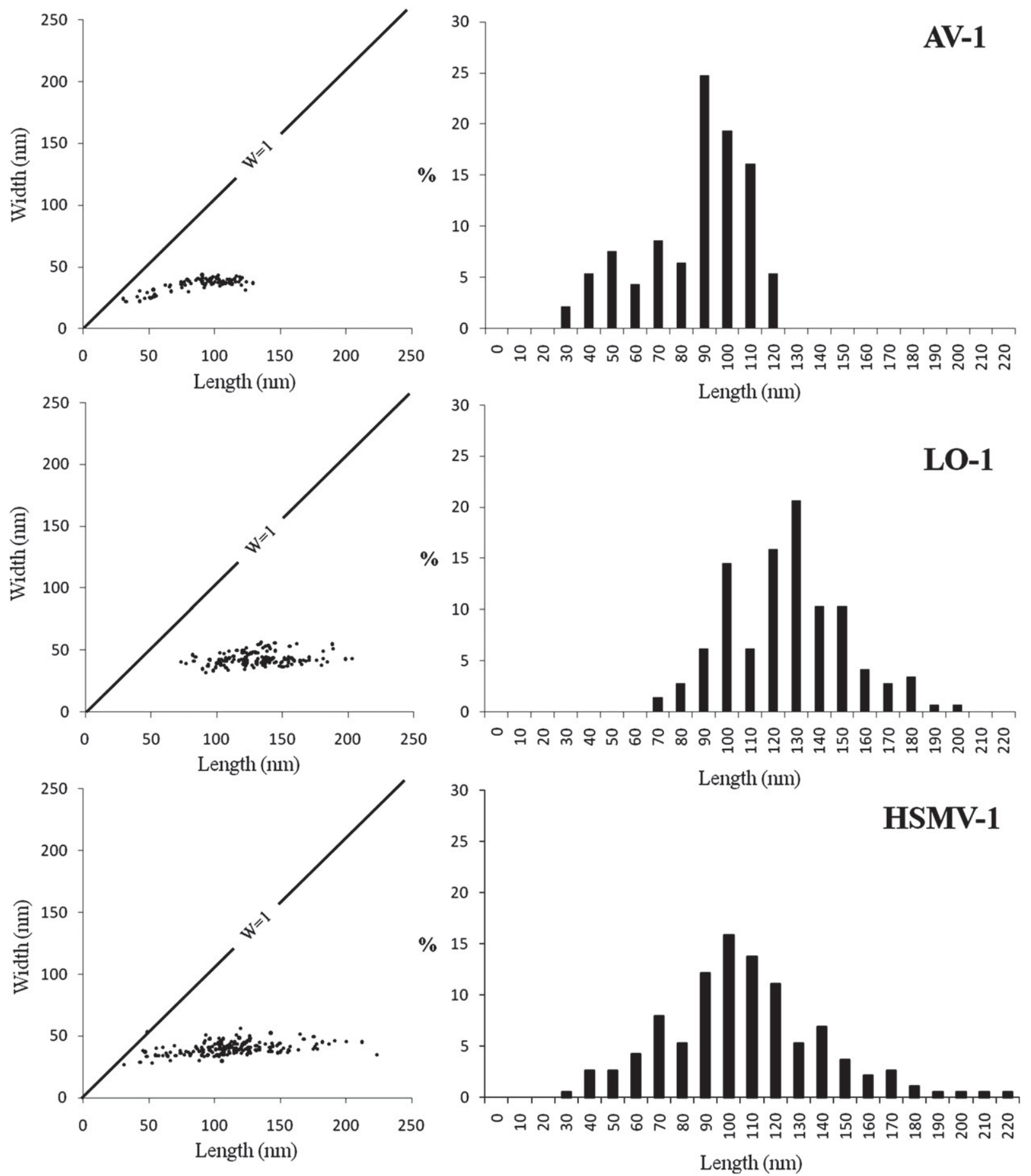

Fig. 10. Width vs. length and crystal length distributions for strain AV-1, Candidatus Magnetoovum mohavensis strain LO-1 and Candidatus Thermomagnetovibrio paiutensis strain HSMV-1 elongated-anisotropic magnetosome crystals ( $n=93$ for AV-1 dts; $n=145$ for LO- $1 ; n=179$ for HSMV- $1 \mathrm{fts}$ ).

crystalline anisotropy is relatively low compared to shape anisotropy in magnetite crystals with dimensions below about $20 \mu \mathrm{m}$, so crystal shape is the most important determinant of the easy and hard magnetic directions (Frankel et al., 2007). Therefore, the orientation of an elongated crystal in a chain is more important than the crystallographic direction to which the axis of elongation is parallel.

The crystal size distributions (CSDs) of magnetosomes in MTB depend on the growth processes of the magnetosome crystals. For most MTB strains affiliated with the Alphaproteobacteria, the shape-factor (width/length) distribution is relatively narrow, indicating proportional growth between width and length. For octahedral and cuboctahedral crystals, the constant of proportionality is close to 1 (e.g., for magnetosome crystals of Magnetospirillum gryphiswaldense strain MSR- 1 on average, $0.91 \pm 0.07(n=147)$ calculated from data in Supplemental Fig. 1), whereas for elongated-prismatic crystals, the constant of proportionality is less than 1 (e.g., for magnetosome crystals of Candidatus Magnetovibrio blakemorei strain MV-1 on average $0.80 \pm 0.09(n=147)$ calculated from data in Supplemental Fig. 1), corresponding to a greater degree of elongation (Arató et al., 2005; Meldrum et al., 1993a, 1993b). For elongated-anisotropic crystals, the maximum of the shape factor distribution was at values $<0.5$ (see Supplemental Fig. S2). A linear relation between width and length occurs for cuboctahedral magnetosomes in M. gryphiswaldense strain MSR-1, and for elongated-prismatic magnetosomes in $\mathrm{Ca}$. Magnetovibrio 
blakemorii strain MV-1 (see Supplemental Fig. S1). In both cases, the linear relationship holds over the entire range of crystal lengths.

The growth of elongated-anisotropic magnetite crystals appears to be more complex than that of the proportional-growth crystals. The growth of magnetosome crystals in ER was proposed to occur in two stages (Isambert et al., 2007; Li et al., 2010; Mann et al., 1987a, 1987b). The nascent cuboctahedral crystals have proportional growth in length and width. Then at a certain size, the crystal commences anisotropic growth along a particular crystal axis while the crystal dimension perpendicular to that axis remains relatively constant. It is interesting that the transition from proportional to anisotropic growth invariably occurs at a crystal length of $40-50 \mathrm{~nm}$ for MTB with elongated-anisotropic magnetosomes. How MTB cause the transition between the two growth mechanisms is unknown. However, the result is a loss of an inversion center in the crystal habit. To our knowledge, lack of an inversion center has never been reported in the habits of geological, or laboratory-produced, magnetite crystals. Therefore, lack of inversion symmetry in mature crystal habits may be a dependable sign of biomineralization (e.g., Schumann et al., 2008).

Although aggregates of nanoscale hematite $\left(\mathrm{Fe}_{2} \mathrm{O}_{3}\right)$ crystals were previously found in some cultures of Desulfovibrio magneticus strain RS-1, the crystals were extracellular (Pósfai et al., 2006). The aggregate magnetosomes found with dts magnetosomes in strain AV-1 are the first reported instance of single magnetosomes formed by multiple magnetite crystals with different but not twin orientations. Perhaps these magnetosomes result from multiple nucleation events in a single magnetosome membrane vesicle with the individual crystallites growing together and the overall shape of the composite determined by constraints imposed by the vesicle.

Finally, it is difficult to think of a growth process that leads to the bent, elongated-anisotropic fts magnetosomes or to understand the adaptiveness, if any, of these habits. One possibility, consistent with finding that each bent magnetosome is a single crystal, is that the elongation bends $60^{\circ}$ from one $<110>$ axis to another of the same form. Because such bending has never been reported in dts crystals, it could mean dts and fts crystals grow by separate processes. The relationship between dts and fts crystal growth will require further study. However, elongated-anisotropic crystals remain robust biomarkers (Arató et al., 2005; Jimenez-Lopez et al., 2010).

Supplementary materials related to this article can be found online at doi:10.1016/j.epsl.2011.10.003.

\section{Acknowledgments}

C.T.L. and D.A.B. were supported by U.S. National Science Foundation grant EAR-0920718. C.T.L. was also the recipient of an award from the Fondation pour la Recherche Médicale FRM: SPF20101220993. U.L and F.A acknowledge partial financial support from Brazilian CNPq, CAPES and FAPERJ.

\section{References}

Arató, B., Szányi, Z., Flies, C., Schüler, D., Frankel, R.B., Buseck, P.R., Pósfai, M., 2005. Crystal size and shape distributions of magnetite from uncultured magnetotactic bacteria as a potential biomarker. Am. Mineral. 90, 1233-1241.
Bazylinski, D.A., Frankel, R.B., 2004. Magnetosome formation in prokaryotes. Nat. Rev. Microbiol. 2, 217-230.

Blakemore, R.P., Frankel, R.B., Kalmijn, A.J., 1980. South-seeking magnetotactic bacteria in the southern hemisphere. Nature 236, 384-385.

Frankel, R.B., Dunin-Borkowski, R.E., Pósfai, M., Bazylinski, D.A., 2007. Magnetic microstructure of magnetotactic bacteria. In: Baeuerlein, E. (Ed.), Handbook of Biomineralization. Wiley-VCH, Weinheim, pp. 127-144.

Hanzlik, M., Winklhofer, M., Petersen, N., 2002. Pulsed-field-remanence measurements on individual magnetotactic bacteria. J. Magn. Magn. Mater. 248, 258-267.

Isambert, A., Menguy, N., Larquet, E., Guyot, F., Valet, J.P., 2007. Transmission electron microscopy study of magnetites in a freshwater population of magnetotactic bacteria. Am. Mineral. 92, 621-630.

Jimenez-Lopez, C., Romanek, C.S., Bazylinski, D.A., 2010. Magnetite as a prokaryotic biomarker: a review. J. Geophys. Res.-Biogeo. 115, G00G03.

Lefèvre, C.T., Abreu, F., Schmidt, M.L., Lins, U., Frankel, R.B., Hedlund, B.P., Bazylinski, D.A., 2010. Moderately thermophilic magnetotactic bacteria from hot springs in Nevada. Appl. Environ. Microbiol. 76, 3740-3743.

Lefèvre, C.T., Frankel, R.B., Abreu, F., Lins, U., Bazylinski, D.A., 2011a. Culture-independent characterization of a novel, uncultivated magnetotactic member of the Nitrospirae phylum. Environ. Microbiol. 13, 538-549.

Lefèvre, C.T., Frankel, R.B., Pósfai, M., Prozorov, T., Bazylinski, D.A., 2011b. Obligately alkaliphilic magnetotactic bacteria from extremely alkaline environments. Environ. Microbiol. 13, 2342-2350.

Li, J., Pan, Y., Liu, Q., Yu-Zhang, K., Menguy, N., Che, R., Qin, H., Lin, W., Wu, W., Petersen, N., Yang, X., 2010. Biomineralization, crystallography and magnetic properties of bullet-shaped magnetite magnetosomes in giant rod magnetotactic bacteria. Earth Planet. Sci. Lett. 293, 368-376.

Lins, U., Keim, C.N., Evans, F.F., Farina, M., Buseck, P.R., 2007. Magnetite $\left(\mathrm{Fe}_{3} \mathrm{O}_{4}\right)$ and greigite $\left(\mathrm{Fe}_{3} \mathrm{~S}_{4}\right)$ crystals in multicellular magnetotactic prokaryotes. Geomicrobiol. J. $24,43-50$

Lins, U., McCartney, M.R., Farina, M., Frankel, R.B., Buseck, P.R., 2005. Habits of magnetosome crystals in coccoid magnetotactic bacteria. Appl. Environ. Microbiol. 71, 4902-4905.

Mann, S., Frankel, R.B., Blakemore, R.P., 1984. Structure, morphology and crystal growth of bacterial magnetite. Nature 310, 405-407.

Mann, S., Sparks, N.H.C., Blakemore, R.P., 1987a. Structure, morphology and crystal growth of anisotropic magnetite crystals in magnetotactic bacteria. P. Roy. Soc. Lond. B Bio. 231, 477-487.

Mann, S., Sparks, N.H.C., Blakemore, R.P., 1987b. Ultrastructure and characterization of anisotropic magnetic inclusions in magnetotactic bacteria. P. Roy. Soc. Lond. B Bio. 231, 469-476.

Matsuda, T., Endo, J., Osakabe, N., Tonomura, A., Arii, T., 1983. Morphology and structure of biogenic magnetite. Nature 303, 411-412.

Meldrum, F.C., Mann, S., Heywood, B.R., Frankel, R.B., Bazylinski, D.A., 1993a. Electron microscopy study of magnetosomes in a cultured coccoid magnetotactic bacterium. P. Roy. Soc. Lond. B Bio. 251, 231-236.

Meldrum, F.C., Mann, S., Heywood, B.R., Frankel, R.B., Bazylinski, D.A., 1993b. Electron microscopy study of magnetosomes in two cultured vibrioid magnetotactic bacteria. P. Roy. Soc. Lond. B Bio. 251, 237-242.

Pikuta, E.V., Hoover, R.B., Bej, A.K., Marsic, D., Whitman, W.B., Cleland, D., Krader, P., 2003. Desulfonatronum thiodismutans sp. nov., a novel alkaliphilic, sulfatereducing bacterium capable of lithoautotrophic growth. Int. J. Syst. Evol. Microbiol. 53, 1327-1332.

Pósfai, M., Moskowitz, B.M., Arató, B., Schüler, D., Flies, C., Bazylinski, D.A., Frankel, R.B., 2006. Properties of intracellular magnetite crystals produced by Desulfovibrio magneticus strain RS-1. Earth Planet. Sci. Lett. 249, 444-455.

Schumann, D., Raub, T.D., Kopp, R.E., Guerquin-Kerne, J.-L., Ting-Di, W., Rouiller, I., Smirnov, A.V., Sears, S.K., Lücken, U., Tikoo, S.M., Hesse, R., Kirschvink, J.L., Vali, H., 2008. Gigantism in unique biogenic magnetite at the Paleocene-Eocene Thermal Maximum. P. Natl. Acad. Sci. U. S. A. 105, 17648-17653.

Thornhill, R.H., Burgess, J.G., Sakaguchi, T., Matsunaga, T., 1994. A morphological classification of bacteria containing bullet-shaped magnetic particles. FEMS Microbiol. Lett. 115, 169-176.

Vali, H., Kirschvink, J.L., 1990. Observations of magnetosome organization, surface structure, and iron biomineralization of undescribed magnetic bacteria: evolutionary speculations. In: Frankel, R.B., Blakemore, R.P. (Eds.), Iron Biominerals. Plenum Press, New York, pp. 97-115. 